\title{
Anelastic Mechanical Behavior Measurement of thin Films Using Novel Beam deflection Method
}

\author{
C.-J. Tong ${ }^{1}$, K.-S. Hsing, ${ }^{1}$, and M.-T. Lin ${ }^{1, a}$ \\ 1 Institute of Precision Engineering, National Chung Hsing University,402 Taichung, Taiwan
}

\begin{abstract}
In this paper a technique developed for studying the anelastic behavior of nano-scale thin metal films on substrate is presented. The test microstructure was designed the triangular cantilever beam and fabricated by the standard C-MOS processes, which can improve stress distribution non-uniform problem and the thickness regime of deposited metal thin film on its surface could reduce to several nanometers. In order to reduce the measure error and calculation complex due to the contact force (like indenter), the driving system was used electrostatic force to making the paddle cantilever beam bend and the deflection of paddle cantilever beam due to the electrostatic force was measured by a capacitance change (Fig. 1). The deflection of the paddle beam can be measured from the capacitance value. A force equilibrium calculate method (include sample compliance force, force due to the film, force due to the gravity and electrostatic force) could determine the stress and strain of the deposited films easily. The anelastic behavior and internal friction study of 200 500 nm Ag \& Al thin film were studied using the dynamic frequency response of the paddle structure generated by electrostatic force under vacuum pressure. The results in loss mechanism show evidences of grain boundary motion and dislocation motion in the film.
\end{abstract}

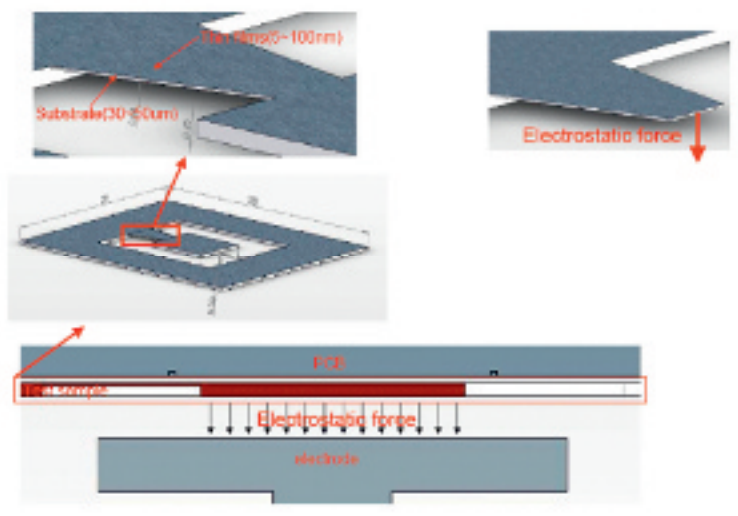

Fig. 1. Schematic of Measurement System 